\title{
动物骨骼表面人工痕迹的三维数字模型及正投影 等值线分析
}

\author{
武仙竹 ${ }^{()^{*}}$, 王运辅 ${ }^{(1}$, 裴树文 ${ }^{(2)}$, 吴秀杰 ${ }^{(2)}$ \\ (1) 重庆师范大学科技考古实验室, 重庆 400047; \\ (2) 中国科学院古脊椎动物与古人类研究所, 北京 100044 \\ E-mail: the168@cqnu.edu.cn
}

2008-06-03 收稿, 2008-12-15 接受

中国科学院知识创新工程重要方向项目(编号: KZCX2-YW-106)、国家自然科学基金(批准号: 40772016)和重庆市教委社科基金重点项目(批 准号: 08JWSK039)资助

摘要 古人类遗址中埋藏的动物骨骼化石与古人类活动密切相关. 动物种类组成、骨骼破碎现 象和骨骼表面痕迹等反映着古人类环境适应、食物选择、食物加工和文化习惯等多方面信息. 目前, 国内对出土骨骼表面痕迹研究非常薄弱. 国际上对骨骼痕迹现象也主要采用肉眼观察、 光学显微镜观察和电子显微镜扫描观察等, 这些方法可以获取大量信息, 但均存在有一定缺 陷. 通过制作痕迹三维数字模型, 对骨骼表面痕迹进行多视角观察和测量, 并对其使用等值线 分析技术, 实现了对痕迹从平面到立体、从静态到动态、从简单数据测量到任意数据测量的研

关键词 古人类 行为模式 骨骼痕迹 三维数字模型 等值线分析 究, 从而有效地分析痕迹性质(痕迹产生原因), 以及产生痕迹的工具类型、刃口形状、工具运 动方式、工具微磨损形态等. 通过对湖北郧西白龙洞古人类遗址出土动物骨骼表面痕迹的实验 研究, 确定了痕迹性质, 而且对产生痕迹的刃形、先后顺序、工作方位及痕迹产生过程中刀形 的微观变化等, 都有较准确的认识. 该实验是人们首次对古人类翟刺性痕迹现象进行专业技术 分析的结果.

古人类演化过程中, 为生存和适应环境, 除发生 了生物学特征的变化外(如脑量增加、身体机能及形 态大小的变化等), 其行为模式也经历了一系列演化. 行为模式反映人类对生存环境的行为适应 (behavior adaptation), 包括制造与使用工具、用火、狩猎等 ${ }^{[1,2]}$. 其中获取动物性肉质蛋白是促进古人类演化进步的 最重要适应性特征之一. 这种行为特征一般是通过 狩猎、宰杀和敲骨吸髓等肉食行为完成. 所以在古人 类遗址发现的动物骨骼中, 保留有人类演化过程中 行为模式的丰富信息. 古人类遗址中的动物种类组 成、骨骼部位、骨骼破碎情况及骨骼表面痕迹等, 是 研究古人类适应环境、食物选择、获取食物的方式以
及工具使用等方面的基本素材 ${ }^{[3]}$. 西方学者对遗址中 骨骼表面的人工痕迹已进行过较多研究, 并取得了 有意义的结果. 如对非洲、欧洲古人类遗址骨骼表面 的切割痕迹进行观察, 提出了痕迹鉴定的一些方法 与标准 ${ }^{[4]}$, 并就原始人类狩猎、屠宰行为和左右手使 用习惯等展开了积极探索 ${ }^{[5 \sim 7]}$. 在西方学者根据骨骼 化石表面痕迹热烈讨论史前人类行为、文化时, 中国 在该方面也取得了一些非常宝贵的成果. 如高星等 人 ${ }^{[8]}$ 通过三峡兴隆洞遗址的研究, 发现该地点有人类 最早有意识的刻划作品.

目前，国内外学者对古人类遗址动物骨骼表面 的痕迹研究, 主要采用肉眼、手持放大镜、体视显微 
镜及电子显微镜等观察方法. 这些方法皆有一定的 作用, 可以提取大量信息, 但也都存在有一些局限性. 如进行肉眼和手持放大镜观察时, 对痕迹不能放大 或放大倍数较小, 满足不了对痕迹微观特征进行分 析, 而且欠缺对观察对象的图像共享. 使用体视显微 镜和电子显微镜工作时, 能够对痕迹放大观察, 并且 在配备摄像镜头等现代光学设备前提下, 可提供图 像资源共享. 但它们所提取的信息主要反映痕迹平 面结构, 在对痕迹的多视角观察、测量和计算方面不 方便使用. 由于现阶段痕迹研究方法均有一定局限, 所以, 在由微观痕迹特征(痕迹运行方向、工具对痕迹 底部与壁面的改造、痕迹产生顺序等方面)分析人类 行为与文化等问题时, 学术界尚缺乏较一致的标准. 因而，在观测技术和手段方面采用新方法进一步提 取骨骼表面痕迹的信息, 对于研究古人类行为特征 及生活方式具有重要意义. 本文通过对骨骼表面痕 迹进行三维扫描, 制作痕迹三维数字模型, 并使用相 关软件实现对痕迹进行多视角 (三维立体)观察、测量 及进行正投影等值线分析. 从而建立一种新的实验 考古学方法, 研究古人类的行为特征与生活方式.

\section{1 研究实验}

\section{1 标本来源与材料特征}

湖北郧西白龙洞古人类遗址曾出土 7 枚直立人 牙齿化石和大量动物化石, 并有石制品等 ${ }^{[9]} .2007$ 年 4 9 月, 中国科学院古脊椎动物与古人类研究所等单 位, 对该遗址进行了再次探察和试掘, 发掘出石制品 和表面带有人工痕迹的化石等 ${ }^{[10]}$. 本文实验标本即 是该次发掘中出土的TN2W2(2): 59 号标本(图 1(a)). 标本是 1 件大型哺乳动物管状骨骨片(化石), 骨片外 表面遗留有 2 处明显的凹痕. 2 处痕迹基本呈并行排 列, 外形近似锐角三角形, 外廓大小相近. 左右间距 约 $4 \mathrm{~mm}$. 最宽处约 $3 \mathrm{~mm}$, 最长约 $8 \mathrm{~mm}$. 痕迹最深点 均在较宽的一端. 为研究方便计, 我们将骨片表面痕

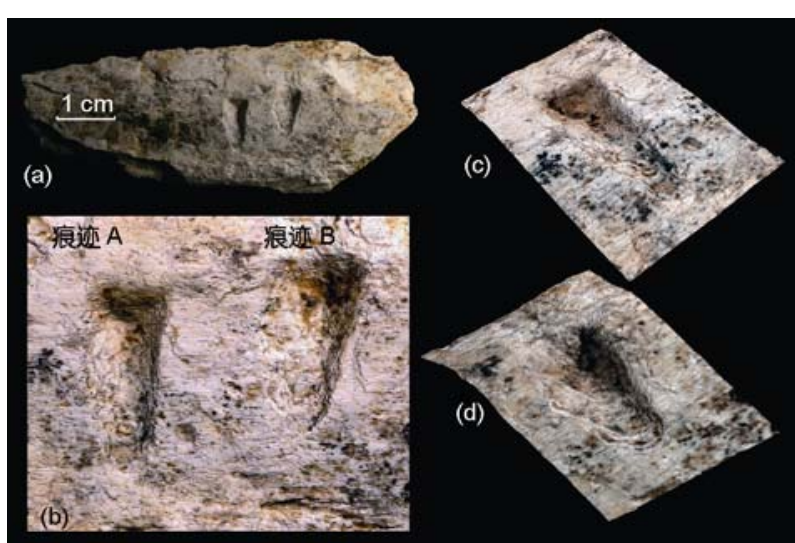

图 1 白龙洞遗址 TN2W2(2): 59 号标本及痕迹

$$
\text { 三维数字模型 }
$$

(a) TN2W2(2): 59 号标本照片; (b) 痕迹 A, B 数字模型的俯视正投 影视图; (c) 痕迹 A 数字模型西南视图; (d) 痕迹 B 数字模型西南 视图

迹按拍摄方向, 左侧凹痕编号为痕迹 $\mathrm{A}$, 右侧凹痕编 号为痕迹 B(参见图 1(b) (d)).

\section{2 三维数字模型的制作与观测}

使用仪器: 日本 Roland 接触式三维扫描仪, 型 号: PIX-30/4. 扫描精度 $0.05 \mathrm{~mm}$. 配套扫描软件 Dr. Prza; 配套使用测量软件 AutoCAD2006.

工作经过：把观测标本置放于扫描仪工作区内. 对需要观测的痕迹 A, B 及相关区域进行扫描. 扫描 过程耗时约 $18 \mathrm{~h}$. 扫描结束后, 将取得的三维数据另 存为 DXF 格式文件, 然后导入 AutoCAD, 生成三维 立体数字模型. 获得痕迹模型后, 我们对其进行了旋 转式多角度观察. 为准确把握数字模型的翻转程度, 我们给模型正面、反面分别设定为不同颜色(图 2). 数 字模型反映, 痕迹 A, B 不仅在骨骼外表面有相近特 征, 而且在模型的背面、侧面也具有相近特征. 为了 能够从数据参数上准确反映出 2 个痕迹的各种观测 特征, 我们对数字模型采用立体图像惯用的“三视 图” 比较法 (以俯视方向的长轴为准, 并使长轴与坐标

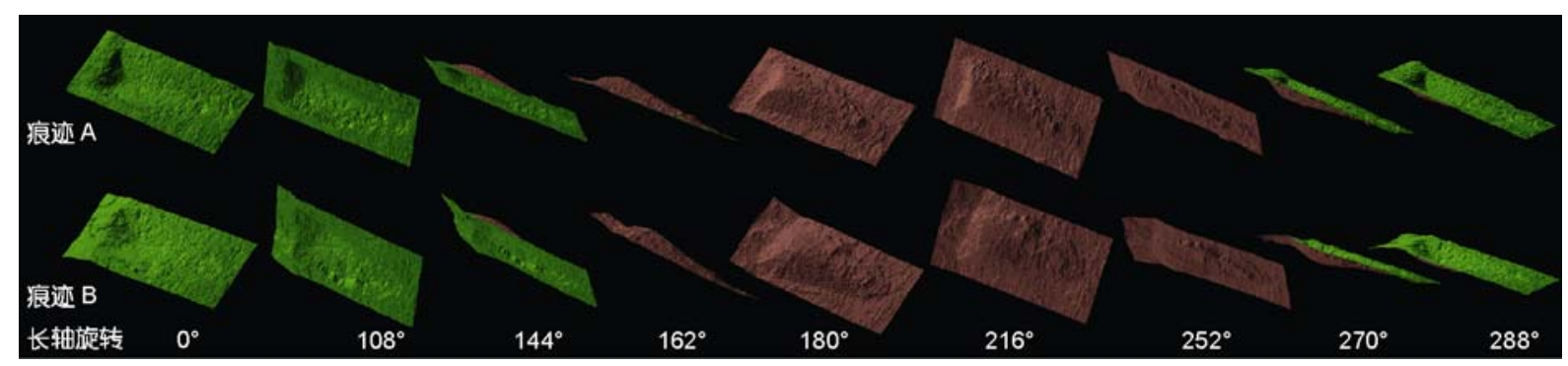

图 2 痕迹三维数字模型的动态对比观察(白龙洞遗址 TN2W2(2): 59 号标本痕迹 A 与痕迹 B) 
$\mathrm{Y}$ 轴平行, 自左往右即获得左视图, 自前往后即获得 前视图), 以便从立体结构上比对 2 个痕迹模型的结 构关系.

观测结果: 在俯视图比对上, 2 个痕迹外廓有位 置基本一致的 7 个转折点(分别命名为 a1 a7 点, b1 b7 点), 底部(痕迹最深处)有位置基本一致的中心 受力点(分别命名为 $\mathrm{a} 0, \mathrm{~b} 0$ 点). 在 AutoCAD工作环境 下, 对各转折点之间的直线距离和转角, 以及中心受 力点和各转角之间的距离分别进行对比(图 3). 在痕 迹 A, B 左视图比对上, 也围绕有 7 个位置基本一致 的转折点(分别命名为 $a 8 \sim a 14, b 8 \sim b 14$, 图 3); 前视图 比对上, 存在有 4 个对应转折点(分别命名为 $\mathrm{a} 15$ a 18 , b15 b18, 图 3).

通过三视图及结构关系对比, 痕迹 A, B 存在有 相同点, 也存在有一些区别. 相同点: 痕迹 A, B 的面 积大小、外廓转折点和受力动力特征基本一致. 痕迹 外廓均有位置基本一致、一一对应的 7 个转折点, 转 折点之间的连线距离相近. 痕迹产生原因的受力特 征, 都是围绕 1 个中心受力点产生 1 个近似长三角形 的深痕. 痕迹的深度也基本一致. 这些特征显示, 痕 迹 $A, B$ 可能是使用同一工具、在连续动作和受力条 件下产生. 区别: 痕迹 A 比痕迹 B 外廓边缘线规则, 外廓转折点之间的连线较直, 壁面略显规整. 痕迹 B 表面比痕迹 A 破损程度略显著. 在左视图底部夹角 和前视图底部夹角上, 痕迹 $\mathrm{A}$ 夹角较小, 痕迹 $\mathrm{B}$ 夹角 明显偏大. 痕迹 $\mathrm{B}$ 底部转折点比痕迹 $\mathrm{A}$ 显得圆滑. 这 些痕迹形态上的微观区别和数据差异, 可能是痕迹 产生先后顺序的反映. 它们暗示产生痕迹 A 时工具 刀口较锐, 而产生痕迹 $\mathrm{B}$ 时刀口已发生变钝和微破 损现象(痕迹 A 先产生, 痕迹 B 后产生).

\section{3 痕迹俯视正投影等值线图观察}

正投影等值线分析技术，是在 Dr. Prza 软件工作 环境下, 利用已经扫描、制作的痕迹三维数字模型, 分别从它们的最深点起始, 向上每间隔一定的微小 差距, 依次截取不同层次的等高面, 直至达到观察对 象的最高点. 然后将获得的全部等高面自动叠加, 按 高度比例尺自动分层调色, 得到被扫描痕迹的正投 影等值线图.

\section{(i ) 等值线围合状况}

痕迹 A: 蓝色系列(500 $\mu \mathrm{m}$ 以下)等值线分别围合 成若干较规整的锐角三角形，绿色等值线围合. 黄色 系列等值线在痕迹上方不围合, 在痕迹下方构成与
蓝、绿等值线相似的转折关系(图 4). 痕迹最深点位 于三角形的短边一侧. 等值线的曲线形状表现出以 最深点为中心的放射状. 放射形状反映出是工具刀 端向下运动时, 挤压骨骼、冲击力四周扩散的结果. 等值线的放射线形状清晰、自然，无叠加现象，显示 该痕迹是受工具作用力一次性产生形成. 等值线的 平面分布现象，反映工具刀端可能是较规整的锐角 三角形. 从痕迹等值线放射状特点分析, 以痕迹长轴 为中线, 离痕迹受力中心点较近的一端应该为痕迹 前方, 离痕迹受力中心点较远的一端为痕迹后方; 离 痕迹受力中心点较近的一侧, 等值线边缘较为平齐、 等值线密度较大，骨骼表面的破损程度略小; 反之， 则等值线分布较为稀疏，等值线放射形状呈较大的 波浪状, 骨骼表面崩损型破损较重.

痕迹 B: 蓝色等值线也分别围合成若干锐角三角 形, 但各层等值线呈三角形转折的角点相对圆滑, 三 角形的各条边均呈外凸的弧线形状. $600 \mu \mathrm{m}$ 以上的 等值线不围合. 黄色系列等值线在痕迹下方构成的 转折关系也与前述俯视观察结果一致. 等值线外廓 规整性与痕迹 A 相比略差, 受力中心点面积变得略 小, 表面崩痕特征比痕迹 A 显著. 其他特征与痕迹 A 基本一致.

痕迹 $A, B$ 的受力动力特征和等值线放射状形态, 反映它们是同一工具在同一动力条件(行为形态)中 产生. 等值线放射状特点反映离中心受力点较近端 为前端(较宽的一端)、距中心受力点较远端为后端(较 窄的一端).

(ii ) 等值线疏密程度与波浪形态. 等值线疏密 程度可指示痕迹壁面的陡直程度, 越密则壁面越陡 直, 越稀疏则越平缓. 等值线波浪形态指示痕迹壁面 的破碎程度, 波浪形态越小, 则壁面的破碎小, 壁面 较规整, 反映刃口锐利、与加工对象的贴合度高. 等 值线波浪起伏大，则壁面破碎显著，说明刃口钝化， 与加工对象的贴合差、间隙大、震动明显. 等值线波 浪形态的规律朝向, 还指示着受力集中点与动力方 向, 有助于认识加工工具的运动规律.

痕迹 $A$ ：等值线波浪形态是以最深点为中心, 以 放射状向外扩张. 最深点右方和上方的等值线紧密, 波浪形态细小，呈细齿状波动; 左方，尤其是下方的 等值线明显稀疏，呈较大的波浪状分布(图 4). 此外, 最深点左下方部分蓝色等值线有明显内收现象, 在 痕迹底部形成有一个瓶颈结构. 说明工具的运动方 


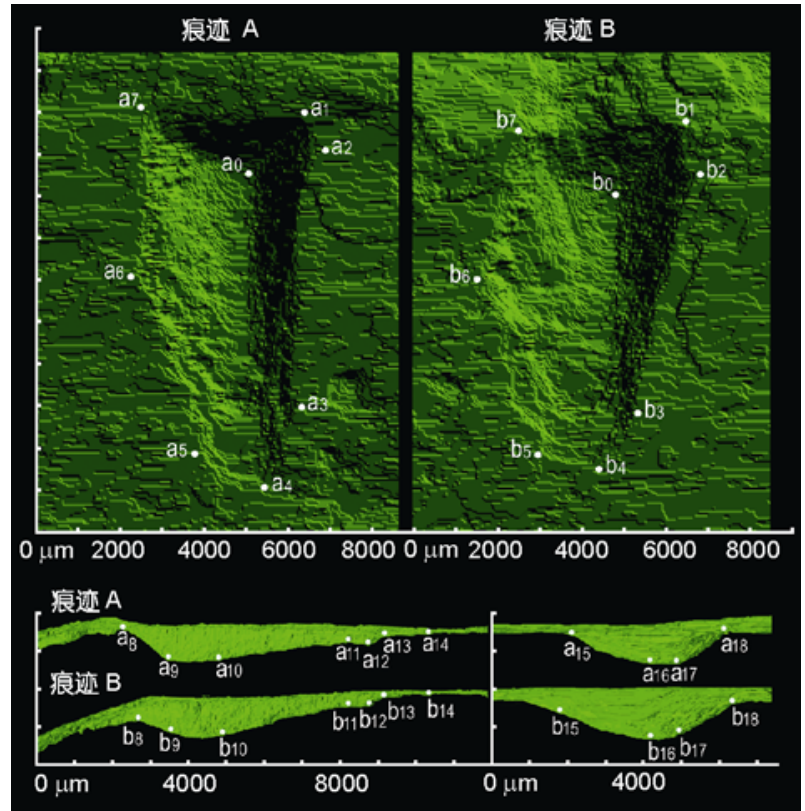

图 3 三维数字模型的计算与测量 (白龙洞遗址 TN2W2(2): 59 号标本)

向，应该是从最深点的上方垂直向下进入. 工具没有 在痕迹底部水平方向上(前后或左右)滑动.

痕迹 B：等值线整体上较痕迹 $\mathrm{A}$ 稀疏，波浪起伏 较大. 但等值线叠加特点和波浪形态的分布关系等,
与痕迹 $\mathrm{A}$ 一致.

痕迹 $\mathrm{A}$ 与痕迹 $\mathrm{B}$ 在结构形态上, 都可分为主体 和次体两大部分. 主体是工具刃口与骨密质直接紧 密接触, 骨密质依刃口形状而产生的形变(即蓝色等 值线围合部分); 次体是工具刃口与骨密质接触不充 分, 甚至不接触, 骨密质因受动力源的间接影响, 而 发生的崩裂性破损(绿色以上等值线部分). 从等值线 围合形状看, 两个痕迹的主体结构是一致的, 均呈三 面雉形体. 但痕迹 B 的三面之间转折关系相对圆滑. 两个痕迹的次体结构也基本一致, 即痕迹下方都有 延伸性崩裂破碎, 而且该部分的轮廓转折关系也基 本一致. 两个痕迹上方也都有崩裂造成的凹陷现象 (即黄色等值线在痕迹上方的不围合现象). 上述现 象, 反映两个痕迹应是使用同一个三棱形尖刀工具 加工, 以相近的力量和运动方式完成的. 加工顺序系 先痕迹 A 后痕迹 B. 工具在加工痕迹 A 之后, 已产生 了一定的钝化现象.

\section{2 讨论与结论}

\section{1 白龙洞遗址实验标本的观测发现}

通过三维数字模型与正投影等值线技术分析, 我们对白龙洞 TN2W2(2): 59 号标本表面痕迹(痕迹 A,

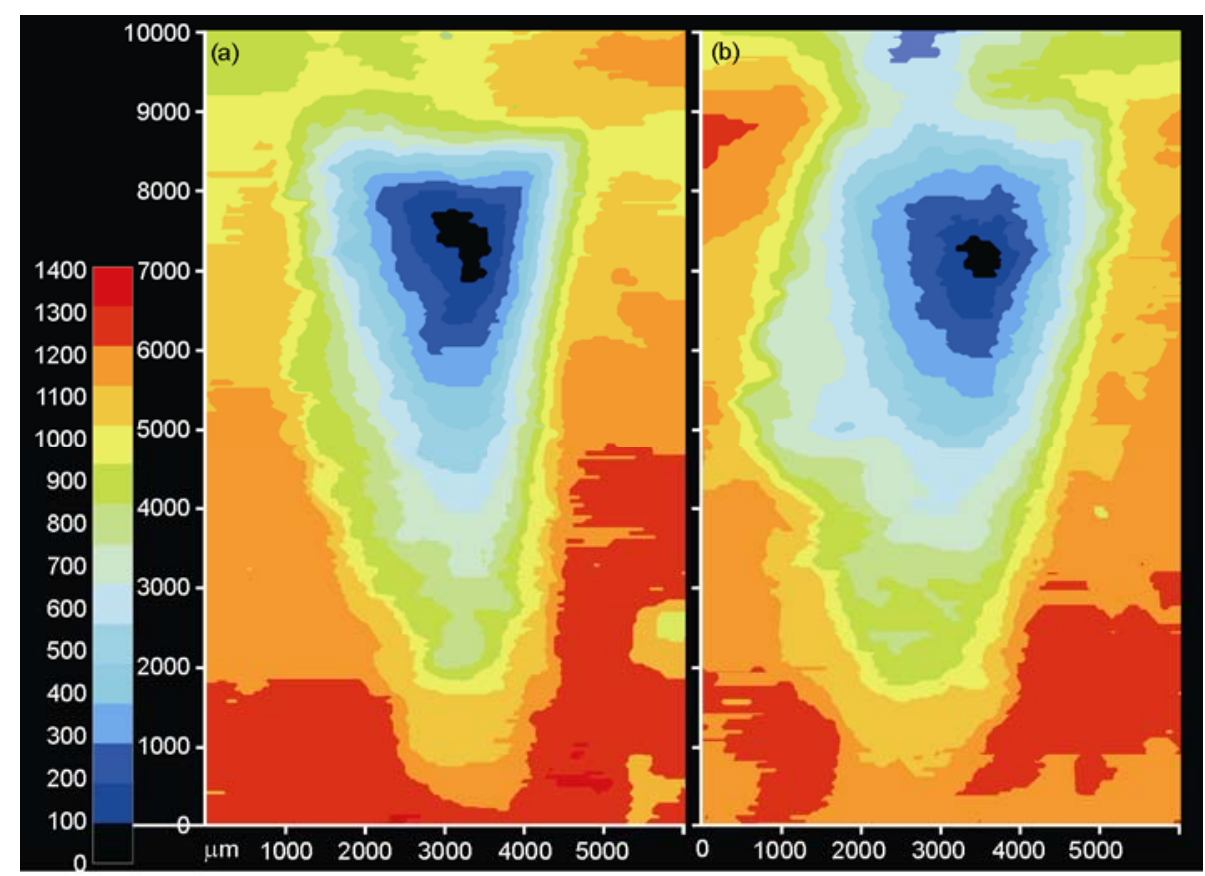

图 4 痕迹正投影等值线分析

(a) 痕迹 $A$; (b) 痕迹 $B$ 
B)的观测取得以下认识.

(i ) 痕迹性质. 该 2 处痕迹是人类使用工具产 生的. 古人类遗址中出土的动物骨骼上, 常能见到很 多种啮齿类和食肉类的啃咬痕迹. 如湖北黄龙洞勋 西人遗址 111 等. 国内外史前考古学者, 目前已对动物 啃咬痕迹有一定认识. 如啮齿类动物啃咬痕迹常常 为上、下对称状, 痕迹比较浅、横断面呈浅 U”形(壁 面与底面呈过渡式转折). 而肉食类啃咬的痕迹, 骨 骼表面常保留有与牙齿特征吻合的牙印 ${ }^{[12]}$. 各种动 物啃咬痕迹的微观性状, 常常是壁面和底面都比较 光滑, 壁面和底面没有清楚的折线状过渡. 在常规观 测技术下，人们对动物啃咬痕迹一般能够做出基本 符合实际的判断. 但由于常规观察提取的主要为痕 迹平面信息特征, 所以在确定人工痕迹性质, 常规观 测技术难以做出准确的肯定性认识. 本文以新的研 究方法, 通过制作痕迹三维数字模型, 在立体、多视 角状态下观测痕迹, 并对其进行等值线特征分析, 达 到准确认定人工痕迹性质的目标. 白龙洞遗址 TN2W2(2)：59 号标本, 其痕迹壁面特征有破损性趐 茬和纤维质断层, 壁面与底面之间有清楚的转折线, 等值线围绕中心受力点呈放射状破损等性状, 与动 物啃咬的表面光滑、由一端向另一端挤压、无清楚壁 面底面转折线的牙齿印痕有明显区别.

(ii ) 古人类行为模式. 古人类在骨骼表面遗留 的痕迹可能由多种行为原因造成, 如砍切、砸击、摔 击、翟刺、刻划、割锯等. 其行为方式有可能是孤立 发生的一次性行为, 也可能是有目的有规划的连续 性行为. 工作对象的方位、工具制作及工具类型选择 等也是行为模式的具体反映. 据实验标本观测, 2 处 痕迹应该是人类使用同一工具在连续动作中产生, 该工具有硬质锐角三角形刀缘特征(与中国旧石器时 代三棱尖状器较相符). 并且痕迹 $\mathrm{A}$ 先产生、痕迹 B 后产生, 属翟刺性行为造成. 工作方位是刀缘长轴与 骨骼长轴横切, 先产生左侧痕迹(痕迹 $\mathrm{A}) 、$ 后产生右 侧痕迹(痕迹 $\mathrm{B})$.

(iii) 痕迹特征中的工具消耗现象. 史前古人类
使用的工具限于材料特性, 每次使用均会产生一定 的磨损、破碎现象(包括石质、骨质、木质工具等). 传 统的史前工具研究中, 对工具连续性使用的消耗现 象难以进行详细研究. 本文根据痕迹数字模型和等 值线分析，发现史前工具在每一次使用过程中均可 观察到较明显的消耗现象, 这为我们根据工具痕迹 研究史前工具生产与工具使用提供了基础.

\section{2 三维数字模型与正投影等值线技术在史前遗址} 痕迹研究中的前景

(i ) 探索多种工具遗留痕迹现象. 在国外, 学 者们对人类刻划类痕迹已进行了一些专门研究 ${ }^{[13,14]}$. 但是, 古人类行为方式和工具使用具有很大的复杂 性. 不同区域古人类的工具类型也有区别(石器文化 的差异性). 本文讨论的使用三棱型锐尖工具(三棱型 大尖状器)对骨骼表面戳刺造成的痕迹现象, 以前还 没有人作过专门研究. 本文对该标本的观察认识, 是 人们对三棱型锐尖工具在骨骼表面遗留痕迹的初步 探索. 该研究方法为我们更多地认识古人类行为与 石器文化提供了条件.

(ii ) 三维数字模型与等值线分析技术在痕迹研 究中的作用. 三维数字模型技术, 在中国科技考古领 域已得到一些成功应用. 如有学者将其用于先秦水 稻植硅体和玉器工艺研究 ${ }^{[15,16]}$. 本文将该技术首次 用于史前工具印痕研究, 并在其基础上进一步做了 等值线技术分析. 以往和本次的实践发现，三维模型 方法在立体图像建造和显微观测方面具有良好作用; 对分析痕迹性质(痕迹产生原因), 以及产生痕迹的工 具类型、刃口形状、工具运动方式、工具微磨损等有 较好作用. 但也需要指出, 三维技术本身尚处在发展 之中, 应用上还有些盲区, 如对器物内腔特征(如器 物内壁凹纹) 就难以获取有效数据 ${ }^{[16]}$. 三维技术能否 全面区分动物啃咬痕迹与人工改造痕迹等复杂现象, 尚待进一步探索和实践. 但从本例及已有研究应用 看, 三维技术在解决痕迹和工具之间的关联性上具 有优势, 它是通过微观痕迹现象去认识人类演化中 行为模式的重要技术途径之一。

参考文献

1 Mcbrearty S, Brooks A. The revolution that wasn't: A new interpretation of the origin of modern human behavior. J Human Evol, 2000, 39: 453-463[ [doi]

2 Henshilwood C S, Marean C W. The origin of modern human behavior: Critique of the models and their test implications. Curr An- 
thropol, 2003, 44: 627-651 [doi]

3 Dominguez R M, Pickering T R. Early hominid hunting and scavenging: A zooarcheological review. Evol Anthropol, 2003, 12: $275-282$ [doi]

4 Timothy G. Bromage, Boyde A. Microscopic criteria for the determination of directionality of cutmarks on bone. Am J Phys Anthropol, 1984, 65: 359-366[doi]

5 Shipman P, Rose J J. Early hominid hunting, butchering, and carcass processing behaviors: Approaches to the fossil record. J Anthropol Archaeol, 1983, 2: 57-98[ [doi]

6 Bromage T G, Castro J M B, Jalvo Y F. The SEM in taphonomic research and its application to studies of cutmarks generally and the determination of handedness specially. Anthropologie, 1991, 3: 163-169

7 Pickering T R, Marschand B H. Cutmarks and hominid handedness. J Archaeol Sci, 2008, 35: 310-315 [doi]

8 高星，黄万波，徐自强，等. 三峡兴隆洞出土 12 15 万年前的古人类化石和象牙刻划. 科学通报, 2003, 48: 2466一-2472

吴汝康，吴新智. 中国古人类遗址. 上海：上海科技教育出版社, 1999. 66-67

武仙竹, 裴树文, 吴秀杰, 等. 湖北郧西白龙洞古人类遗址初步观察. 人类学学报, 2009, 28: 1-14

武仙竹. 勋西人-黄龙洞遗址发掘报告. 北京: 科学出版社, 2006. 1-272

Norton C J, 张双权, 张乐, 等. 上/更新世动物群中人类与食肉动物“印记的识别”. 人类学学报, 2007, 26: 183-192

Shipman P, Rose J J. Cutmark mimics on modem and fossil bovid bones. Curr Anthropol, 1984, 25: 116 - 117 [doi]

4 Cook J. The application of scanning electron microscopy to tapnonomic and archaeological problems. In: Rose D A, ed. Studies in the Upper Palaeolithic of Britain and Northwest Europe, British Archaeological Reports. London: Oxford, 1986. 143-163

15 吴妍，姚政权，龚明，等.三维图像重建在植硅体研究中的应用. 农业考古, 2006, 1: 61-64

16 杨益民，郭怡，谢尧亭，等. 西周倗国墓地绿松石珠微痕的数码显微镜分析. 文物保护与考古科学, 2008, 20: 46一 49

\section{《中国科学 D 辑: 地球科学》 第 39 卷 第 6 期 目次}

粤北存在早侏罗世的岩浆活动一来自霞岗杂岩 SHRIMP 锆石 U-Pb 年代学的证据

余心起 狄永军 吴洤国 张达郑勇 代堰锫

681

华北克拉通东南缘古元古代变质和岩浆事件的锆石 SHRIMP U-Pb 年龄

·郭素淑 李曙光

694

马鞍桥金矿床中香沟岩体锆石 U-Pb 定年、地球化学及其与成矿关系研究

. 朱赖民 张国伟 李 牶 郭 波 弓虎军 康 磊 吕拾零

塔里木盆地下古生界白云石微区 REE 配分特征及其成因研究……

王小林 金之钧 胡文瑄 张军涛 钱一雄 朱井泉 李 庆

柴达木盆地三湖地区产甲烷作用仍在强烈进行的地球化学证据.

帅燕华 张水昌 苏爱国 王汇䑣 蔡报元 王 慧

地球磁层对磁云边界层的大尺度响应分析一个例研究

·左平兵 魏奉思 冯学尚 李汇军

南海西北部末次盛冰期以来的古海岸线重建

.姚衍桃 Jan Harff Michael Meyer 詹文欢

基于 ENVISAT ASAR 数据的水稻估产方案 .中双和 杨沈斌 李秉柏 谭炳香 李增元 Le Toan Thuy

复杂地形下黄河流域月平均气温分布式模拟 曾 燕 邱新法 何永健 施国萍 刘昌明

2008 年初江南冻雨过程的湿大气锋生

钱维宏 符娇兰

西北太平洋海气界面湍流热通量低频振荡强度的特征 李 根 任保华 郑建秋 王 俊 